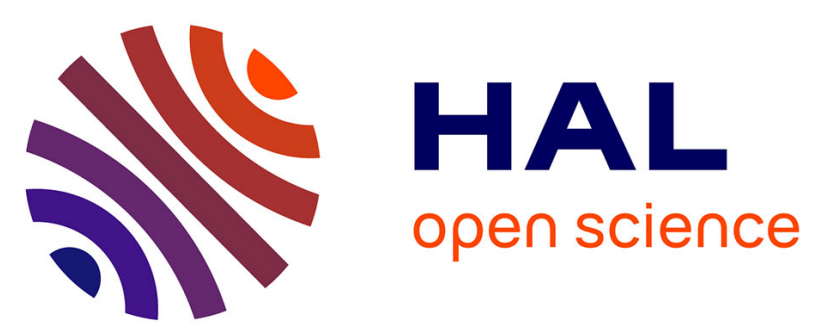

\title{
Resolution and computational strategy in wideband multiphoton microscopy illustrated with muscle imaging
}

Ali Abjaghou, Claire Carrion, Laetitia Magnol, Claire Lefort

\section{To cite this version:}

Ali Abjaghou, Claire Carrion, Laetitia Magnol, Claire Lefort. Resolution and computational strategy in wideband multiphoton microscopy illustrated with muscle imaging. Optics and Photonics for Advanced Dimensional Metrology, Apr 2020, Online Only, France. pp.34, 10.1117/12.2555048 . hal-02566517

\section{HAL Id: hal-02566517 https://hal.science/hal-02566517}

Submitted on 7 May 2020

HAL is a multi-disciplinary open access archive for the deposit and dissemination of scientific research documents, whether they are published or not. The documents may come from teaching and research institutions in France or abroad, or from public or private research centers.
L'archive ouverte pluridisciplinaire HAL, est destinée au dépôt et à la diffusion de documents scientifiques de niveau recherche, publiés ou non, émanant des établissements d'enseignement et de recherche français ou étrangers, des laboratoires publics ou privés. 


\title{
Resolution and computational strategy in wideband multiphoton microscopy illustrated with muscle imaging
}

\author{
Ali Abjaghou ${ }^{\mathrm{a}, \mathrm{b}}$, Claire Carrion $^{\mathrm{b}}$, Laetitia Magnol ${ }^{\mathrm{c}}$, Claire Lefort*a $^{* \mathrm{a}}$ \\ ${ }^{a}$ CNRS UMR 7252, XLIM Research Institute, Université de Limoges, France \\ ${ }^{b}$ BISCEm, Microscopy core Facility Université de Limoges, France \\ ${ }^{c}$ GAAMA Lab, University of Limoges, France
}

\begin{abstract}
Multiphoton microscopy (MPM) is an approach now well established in biomedical sciences, especially thanks to its excitation spectrum in the near infrared range (NIR). The simultaneous imaging of numerous of these substances imposes the use of a wideband excitation spectrum, indispensable in the case of in vivo and in live imaging or for detecting phenomena at video rates. A unique spectral bandwidth, covering the range between 750 and $1000 \mathrm{~nm}$ has been recently demonstrated and has made emerging a simplification in MPM: the excitation system is now no longer an lock for generating multiphoton images of numerous fluorophores. But such a solution might be highly sensitive to chromatic distortions and diffraction limit which might result in detrimental effects on image quality and especially on resolution performance. This question is at the core of the current presentation. A point-spread function (PSF) estimation is realized with a standard computational tool. Our experimental strategy has shown two interesting points. First, the resolution is preserved in the lateral plan (xy) regardless of the excitation procedure chosen. Second, a significant deterioration of the resolution is observed in the axial direction $(\mathrm{z})$, with a factor 4 between the best resolution obtained with a standard imaging procedure and the worst one obtained with the wider spectral bandwidth. Starting with this result, the role of a computational solution of image reconstruction is highlighted for reducing the gap observed in axial resolution between standard and wideband excitation solution of MPM. The illustration of the interest of a large spectral bandwidth of excitation is then shown on a mouse muscle sample presenting 3 fluorophores having a spectral bandwidth of excitation spread along $300 \mathrm{~nm}$. This set of experiments illustrates the impact of chromatic distortions and diffraction limit on the deterioration of resolution. As a conclusion, a basic protocol for image reconstruction is used in order to highlight the interesting level of improvement of the visual image quality generated by a standard computational image restoration.
\end{abstract}

Keywords: Multiphoton microscopy, biomedical imaging, large band excitation strategy, resolution evaluation

*claire.lefort@xlim.fr

\section{INTRODUCTION: CONTEXT OF THE STUDY}

\subsection{Interest of an ultrawide band laser system in multiphoton microscopy for biomedical imaging}

\footnotetext{
Multiphoton microscopy (MPM) is a microscopy method now well established in biomedical imaging sciences [1]. This success results from the excitation strategy based on a spectrum spread in the near infrared range (NIR). NIR of excitation is one of the less dangerous wavelengths for imaging living organisms with an optical method. Such a strategy opens to in vivo imaging. NIR of excitation is less absorbed and scattered by living sample which can become transparent to those NIR excitation wavelengths. In some situations, when the sample imaged is composed by the adapted substances, this imaging method benefits from label-free imaging properties due to the presence of autofluorescent substances or of noncentrosymmetric assembly identified inside the biological sample imaged [2, 3]. These specific probes can generate optical signals detectable without any preliminary labeling preparation [4]. With an adapted laser technology such as a mode-locked titanium-doped sapphire laser (Ti: Sa), two multiphoton processes are mainly available simultaneously: two-photon fluorescence (TPF) and second harmonic generation (SHG) [5, 6]. Complementary modules such as optical parametric oscillators (OPO) or optical parametric amplifiers (OPA) can spread
} 
spectral ranges from $500 \mathrm{~nm}$ until $2.5 \mu \mathrm{m}$. Such excitation ranges give access to three-photon processes: three-photon fluorescence (ThPF) and third harmonic generation (THG) [7]. The price of such a technology and the sequential aspect of the imaging property could be asked; the resulting answer does not play in favor to a choice orientated to these technologies. In that context, the interest of ultrawide band laser systems (UWLS) in MPM has been highlighted about ten years ago [8]. The simultaneous imaging of numerous of these substances imposes the use of a wideband excitation spectrum, indispensable in the case of in vivo and in live imaging or for detecting phenomena at video rates [9, 10]. We have recently demonstrated that a wideband laser system is the exclusive solution allowing the simultaneous imaging of many fluorescent substances regardless their provenance in life sciences: synthetized chemical substances or small animals [9]. This system does not require any modification of excitation parameters: central wavelength, spectral bandwidth and average power. A unique spectral bandwidth, covering the range between 750 and $1000 \mathrm{~nm}$, has made emerge a huge simplification in MPM: the excitation system is now no longer an instrumental lock for generating multiphoton images composed by the fluorescent signals from numerous fluorophores presenting an excitation spectrum spread along bandwidths larger than the $10 \mathrm{~nm}$ of the Ti: Sa excitation systems. But such a solution might be highly sensitive to the impact of diffraction limit and also to chromatic distortions which might result in detrimental effects on image quality and especially on resolution performance.

\subsection{NIR, resolution, PSF evaluation and role of computation strategies in MPM}

Resolution in optical microscopy is one of the physical parameters defining the quality of the system. The evaluation of resolution amounts to estimate the ability of the system to preserve a Dirac signal from its emission (sample plan) to the detection (imaging plan). In optical microscopy, due to optical aberrations and diffraction limit, a Dirac signal is always transformed in a Gaussian signal. The shortest width of the Gaussian is associated to the best quality of the microscope system. Such a characterization results in the estimation of the transfer function of the system, also named Point Spread Function (PSF) estimation. In that context, many optical solutions of super-resolution microscopy methods have been developed [11]. These sophisticated solutions are all based on a complex optical or chemical strategy and result in very attractive performance in terms of resolution: until few nanometers in the optical plan and until few tens of nanometer in the optical axis. One day, maybe the single atom will be imaged with an optical method! But these attractive results are always obtained at the price of a very complex and expensive instrument, never turn-key, which is not possible to use in vivo or in live. Moreover, 3D imaging of complex structures become unattainable and requires clarified structures for their imaging in millimeter-scale [12].

Two physical phenomena occurring simultaneously are mainly responsible to the image deterioration and associated to a damaged resolution. First, the diffraction limit is an unavoidable phenomenon. The more the excitation spectrum is centered far in the NIR, the worst the resolution is. Moreover, when a large spectral bandwidth is employed in optical microscopy, chromatic aberration, a harmful physical phenomenon, is exacerbated and causes a worst resolution. In that context, many computational strategies for improving resolution performance in optical microscopy have appeared and are currently established or in progress $[13,14]$.

The computational strategies developed for optical microscopy are based on the PSF evaluation. The best performance in image restoration is strongly dependent on the reality of PSF evaluation. When the PSF is correctly known thanks to an experimental measurement associated to a correct mathematical modeling, or thanks to a correct theoretical estimation, the contribution of the PSF can be removed to the image thanks to an adapted deconvolution strategy [15]. The software Huygens, a commercial system is using this computational strategy for improving visually the image quality.

Considering that the PSF is a fixed property of a single microscopic measurement linked with the diffraction limit, in the situation of MPM coupled with an UWLS, the diffraction limit becomes a parameter of the first importance. Diffraction limit is an unalterable physical principle determining the resolution properties in optical microscopy. In next section we are presenting the estimation of the impact of the wide spectral bandwidth of excitation in UWLS involved in MPM experiments. 


\section{EXPERIMENTAL PROTOCOLE}

\subsection{Experimental setup of MPM}

A multiphoton microscope from Olympus (BX61WI) is used for all of these experiments. Figure 1 illustrates the resulting system. The excitation source can be switched between a standard Ti: Sa laser source and a commercial supercontinuum laser system which delivers an excitation spectrum spread between $500 \mathrm{~nm}$ and $2.5 \mu \mathrm{m}$. The excitation beam is spectrally filtered thanks to a combination of optical filters. A scanning device generates a local excitation indispensable for producing an image point by point. The detection is realized by two photomultiplier tubes which can collect simultaneously photons emitted from the object plan.

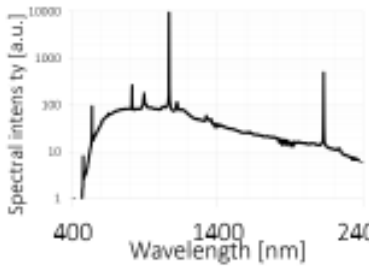

Large band laser source
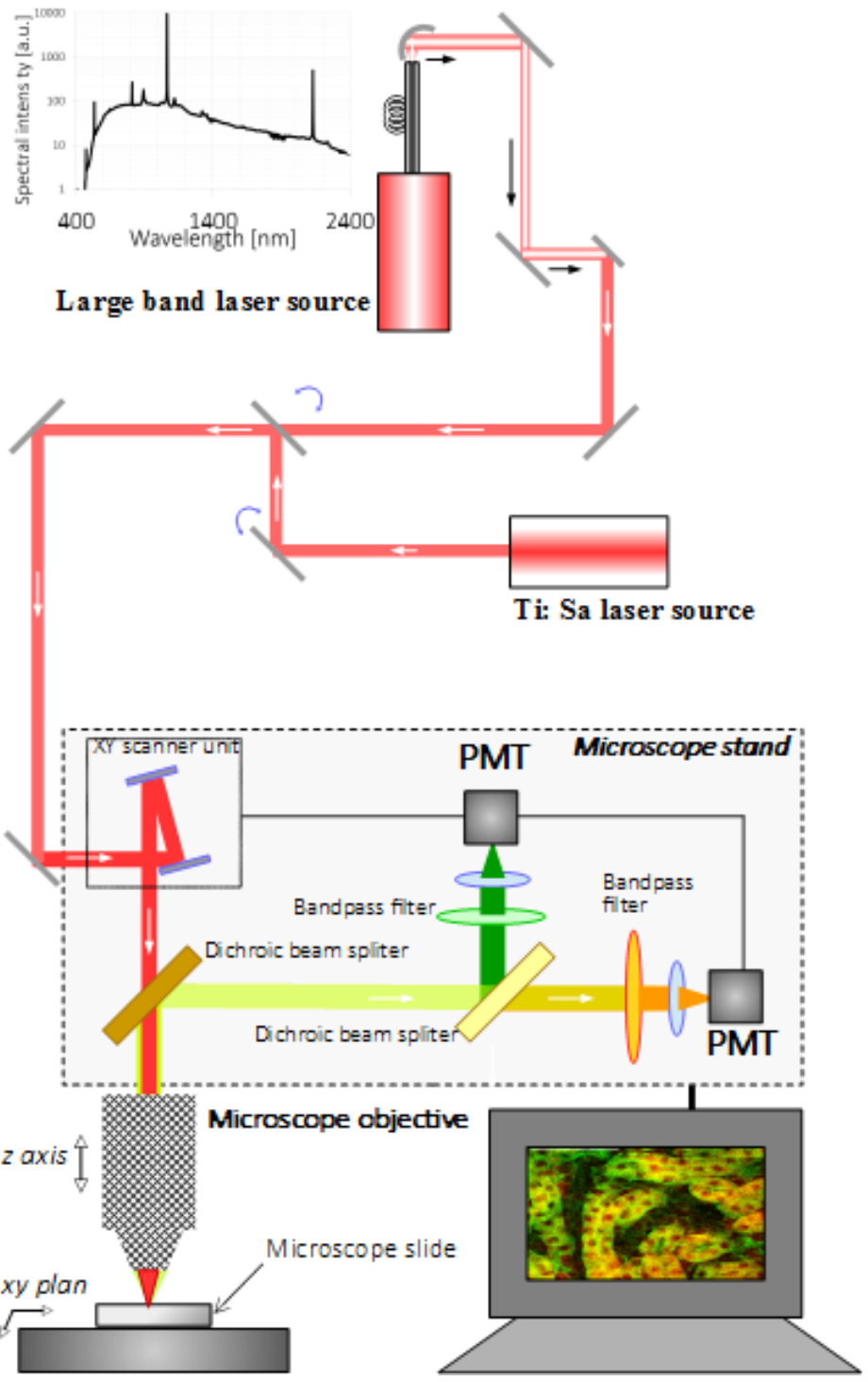

Figure 1. Experimental setup involved for the estimation of the impact of the use of an UWLS in MPM on the resolution performance. A standard protocole for estimating the resolution in optical microscopy is applied. The critical parameter of the optical plan (xy) and optical axis (z) are defined. 


\subsection{Microspheres involved for estimating the resolution of MPM under an UWLS excitation strategy}

A slice of microspheres from Invitrogen is employed. This microscope slice is especially dedicated to the estimation of resolution in conditions of confocal laser scanning microscopy methods. Such a protocol is now translated and adapted to our multiphoton microscope device. Microspheres with a diameter of $0.2 \mu \mathrm{m}$ are used. Two colors are mainly employed for this characterization: green and orange microspheres. In that aim, our first experiment consist in measuring the twophoton excitation spectrum of the microspheres. Figure 2 presents the resulting excitation spectrum for the green microspheres.

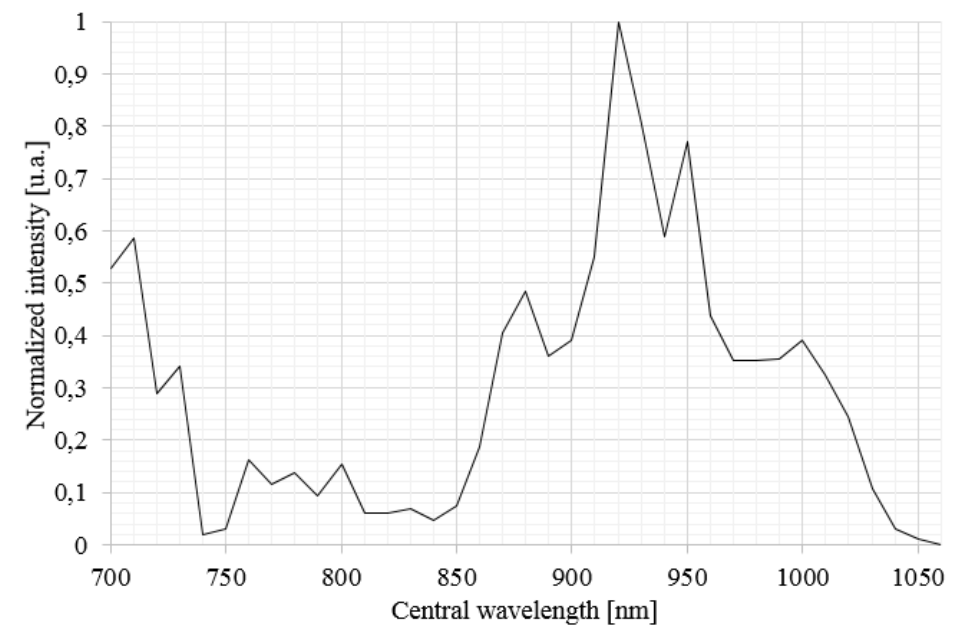

Figure 2. Two-photon excitation spectrum of green fluorescent microspheres employed for the estimation of the imact of an UWLS in the resolution performance in MPM.

The two-photon excitation spectrum for the microspheres must be studied before starting the experiments concerning resolution estimation. Indeed, this excitation spectrum is split in two main part. For an excitation between 700 and 750 $\mathrm{nm}$, and considering the high peak-power delivered by the pulsed lasers, the resulting emission might mostly results to a single photon absorption process. Thus, this part of the excitation spectrum is not considered. Then, for the excitation spectrum spread between 750 and $1050 \mathrm{~nm}$, the absorption efficiency is unequally distributed. By consequence, the estimation of the PSF is evaluated by mastering the average power of the excitation system resulting in the generation of the fluorescence signal all at the same value, meaning by considering fixed parameters of the detection system (photomultiplier tube: fixed voltage, gain and offset all along the experiments).

\subsection{Microspheres image acquisition and computational strategy}

For the different central wavelengths of excitation and spectral bandwidth of excitation, ten microspheres are imaged for each set of experiments. Such a protocol delivers a statistically representative imaging result. Each microsphere is imaged with a voxel dimension of $50 \mathrm{~nm} \times 50 \mathrm{~nm} \times 100 \mathrm{~nm}$. The resulting voxels are then analyzed thanks to a commercial computational strategy named Huygens from SVI (https://svi.nl/Huygens-Software). The PSF estimation is calculated with a Richardson-Lucy algorithm and 40 iteration are involved for each measurement. Then, the microsphere image is deconvolved by itself. Figure 3 shows the resulting image representing the intensity profile generated in standard MPM condition (with a Ti: Sa excitation). The resolution is especially measured in the optical plan thereafter named $x y$ plan or $x y$, and in the optical axis, thereafter named $x z$ or $y z$ or $\mathrm{z}$ plan. 

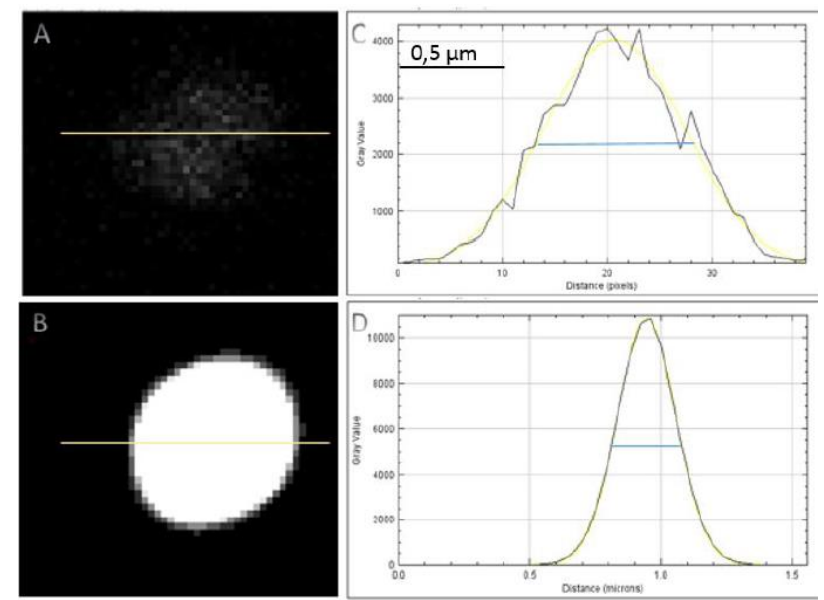

Figure 3. 2D slice of fluroescence intensity microscophere generate with the standard Ti: Sa system recorded at the maximum width of the microsphere on the image. A. Raw image. C. Intensity profile associated to the raw image. B. Image recorded after a deconvolution process. D. Intensity profile associated to the processed image. The Gaussian profile of the PSF appears after the computationnal processing of the raw image.

\section{RESOLUTION ESTIMATION AND IMPACT OF EXCITATION CENTRAL WAVELEGTH AND BANDWIDTH}

\subsection{Impact of the central wavelength of excitation on the resolution: effect of diffraction limit}

Table 1 presents the resulting PSF evaluation with green and orange microspheres when an excitation process is involved with a standard $\mathrm{Ti}$ : Sa excitation system. Two colors of microspheres are used for this setup: green and orange microspheres. The PSF is estimated for raw images. Then, a deconvolution is calculated considering the deconvolution of the microsphere by itself. The resulting PSF is measured and a new resolution value is calculated.

Table 1. PSF estimations for green and orange microspheres. Raw PSF and PSF after deconvolution are measured.

\begin{tabular}{|l|cc|cc|}
\hline \multicolumn{1}{|c|}{ Microsphere } & \multicolumn{2}{c|}{ Raw PSF } & \multicolumn{2}{c|}{ After deconvolution } \\
Green & PSF xy $(\boldsymbol{\mu m})$ & PSF $\mathbf{z}(\boldsymbol{\mu m})$ & PSF xy $(\boldsymbol{\mu m})$ & PSF $\mathbf{z}(\boldsymbol{\mu m})$ \\
Orange & 0.58 & 1.82 & 0.31 & 0.78 \\
\hline
\end{tabular}

Many information are extracted from Table 1. First, as expected, the PSF obtained for the longer wavelengths is worse than for the shortest wavelengths, especially in the optical axis. This difference is visible regardless the raw or the deconvolved image of the PSF. The deconvolution procedure highlights an optimization of the resolution of the microscopy device by $60 \%$ for green and orange microspheres in z axis. An existing optimization of less than $50 \%$ is 
observed in the optical plan xy. Secondly, between the green and orange microspheres, the difference of resolution equals less than $5 \%$ in the optical plan xy regardless after or before the deconvolution procedure.

The conclusion that can be extracted from such a table of measurement, with a very standard protocol is that

$\rightarrow$ No consideration about the use of a deconvolution strategy for resolution improvement

- The resolution difference is very small between green and orange emission wavelengths

- A significant difference of resolution exists in the optical axis (z) between green and orange emission wavelengths

$\rightarrow$ Using a deconvolution calculation for estimating the resolution

- In the optical plan (xy), the resolution is significantly optimized with about $50 \%$

- In the optical axis (z), the resolution is greatly optimized with about $60 \%$

The diffraction limit does not impact significantly the resolution of the MPM system in the optical plan (xy) regardless the use of a deconvolution procedure or not. As expected, the impact of diffraction limit becomes huge in the optical axis. A computational deconvolution optimizes this last parameter but does not gives access to the same level of resolution performances than in the optical plan.

\subsection{Impact of the spectral bandwidth of excitation on resolution: effect of chromatic aberrations}

The spectral bandwidth of the supercontinuum laser source is centered at $900 \mathrm{~nm}$ and is spread between 750 and 1000 $\mathrm{nm}$. The average power is kept constant all along the experiment. For the comparison, the measurement is also realized thanks to the standard Ti: Sa excitation strategy. Green microspheres are employed in that aim. Table 2 concatenates the resulting resolution estimations.

Table 2. PSF estimations for green microspheres with a Ti: Sa excitation strategy and with a supercontinuum excitation strategy. Raw PSF and PSF after deconvolution are measured.

\begin{tabular}{|l|cc|cc|}
\hline & \multicolumn{2}{c}{ Raw PSF } & \multicolumn{2}{c|}{ After deconvolution } \\
Excitation strategy & PSF $\mathbf{x y}(\boldsymbol{\mu m})$ & $\mathbf{P S F} \mathbf{z}(\boldsymbol{\mu} \mathbf{m})$ & PSF $\mathbf{x y}(\boldsymbol{\mu m})$ & PSF $\mathbf{z}(\boldsymbol{\mu m})$ \\
Ti: Sa & 0.55 & 1.83 & 0.29 & 0.34 \\
Supercontinuum & 0.72 & 4.31 & 0.34 & 2.17 \\
\hline
\end{tabular}

The impact of the spectral bandwidth of excitation is strongly linked with the effect of chromatic aberrations on the resolution performance of an optical microscope and especially in the case of a multiphoton microscope. The effect of chromatic aberrations is not significant for the resolution in the optical plan (xy). The effect of chromatic aberrations is significant for the axial resolution where a factor 2.4 increases the resolution of raw images. In case of deconvolution procedure involved, the effect of a wide spectral bandwidth of excitation degrades the resolution performance with a factor 6.4 . 


\section{ILLUSTRATION OF THE INTEREST OF A COMPUTATIONAL STRATEGY FOR THE OPTIMISATION OF IMAGES GENERATED IN MPM WITH AN UWLS}

Now, we illustrates the interest of an ultrawide band laser system in MPM for biomedical imaging. The sample imaged is constituted by an EDL muscle sample resected from a 1-year-old wild mouse. The muscle slice is resected orthogonally to the muscle axis defined from tendon to tendon. By consequence, the muscle fibers appearing at the image are constituted by bundles of fibers. The sample is labeled with three fluorophores. Frist laminin is labeled with Alexa Fluor 633 whose two-photon excitation spectrum is centered at $995 \mathrm{~nm}$. Then, glycolytic fibers are labeled with Alexa Fluor 546 and finally, oxidative fibers are labeled with Alexa Fluor 488. The two-photon excitation spectrum of these two last fluorophores are respectively centered at 880 and $750 \mathrm{~nm}$.

With such an example, the interest of an ultrawide band excitation system takes all its sense: a single shot of excitation generates 3 signals simultaneously and is associated to 3 independent images of 3 different constituents of the same sample. Now, we illustrate the resulting image and its comparison with the same image generated with the three excitation wavelengths coming from the Ti: Sa and requiring 3 different acquisitions. Moreover, thanks to the PSF characterization recorded for each experiment, the impact of computational deconvolution proposed by Huygens software is realized. Figure 4 gathers all the images.
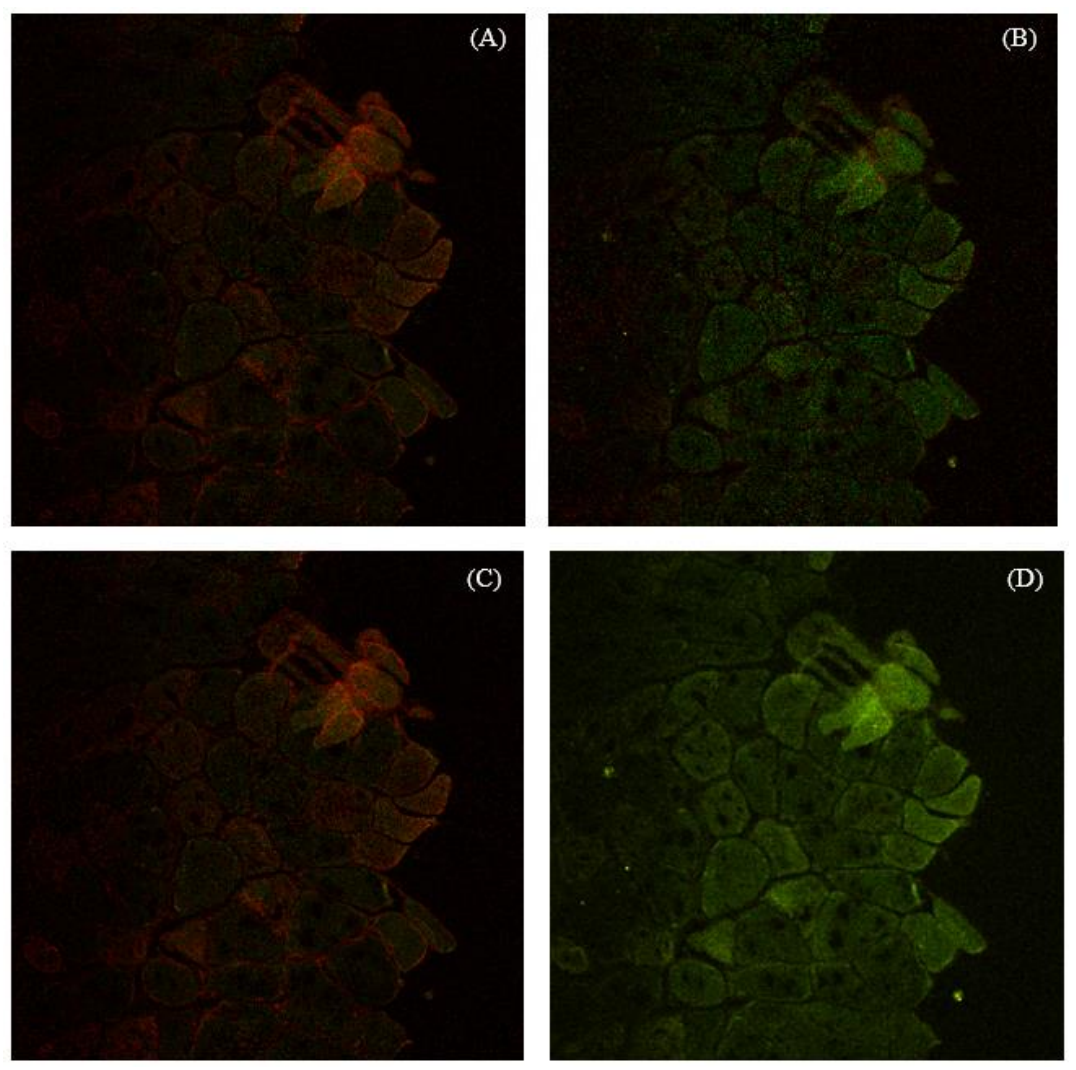

Figure 4. Multiphoton images of bovine pulmonary artery endothelial cells. All the detection parameters are kept constant during all the procedure of image, regardless the excitation protocole. A. Standard multiphoton images with a Ti: Sa system centerd at $750 \mathrm{~nm}$, then $880 \mathrm{~nm}$ and then $995 \mathrm{~nm}$ with a constant average power. B. Multiphoton images obtained with a supercontinuum excitation spread between 750 and $1000 \mathrm{~nm}$. A single shot of excitation is require for the excitation protocole where no modification of the excitation process is required. C. Ti: Sa images of the mouse muscle after a deconvolution process (Huygens, SVI). D. Supercontinuum image of the mouse muscle after a deconvolution process (Huygens, SVI). 


\section{CONCLUSION}

The interest of an ultrawide band laser excitation strategy opens the possibility to image simultaneously numerous fluorophores and especially fluorophores from endogenous origin. The limitation due to the use of a large spectral bandwidth of excitation is linked to the resolution performance. Indeed, such a parameter is highly sensitive to chromatic aberrations and diffraction limit. Here, we have highlighted that the diffraction limit does not impact significantly the resolution performances of a multiphoton microscope. However, the chromatic effect has a high impact on the resolution performance. This last parameter can be compensated by computational strategies whose level of quality highly depends on their ability to estimate correctly the point spread function of the system.

\section{REFERENCES}

[1] J. Mertz, "Introduction to Optical Microscopy", Roberts \& Company Publishers (2009).

[2] J. R. Lakowicz, "Principles of Fluorescence Spectroscopy", 3rd edn, Berlin: Springer (2006)

[3] B. R. Masters, P. T. So, "Handbook of Biomedical Nonlinear Optical Microscopy", Oxford University Press, 2008, 896 pages. ISBN 978-0-1951-6260-9

[4] F.-J. Kao, "The Use of Optical Parametric Oscillator for Harmonic Generation and Two-Photon UV Fluorescence Microscopy", Microscopy Research and Technique, 63, 175-181 (2004)

[5] C. Lefort, "Laser sources in multiphoton microscopy: Overview and optimization", Proceedings of SPIE - The International Society for Optical Engineering, 106770 V (2018)

[6] V. Marx, "It's free imaging - label-free, that is", Nature Methods, 16, 1209-1212 (2019)

[7] L. Abdeladim, K. S. Matho, S. Clavreul, P. Mahou, J.-M. Sintes, X. Solinas, I. Arganda-Carreras, S. G. Turney, J. W. Lichtman, A. Chessel, A.-P. Bemelmans, K. Loulier, W. Supatto, J. Livet, E. Beaurepaire, "Multicolor multiscale brain imaging with chromaticmultiphoton serial microscopy", Nature Communication, 10, (2019)J.

[8] A. Palero, V. O. Boer, J. C. Vijverberg, H. C. Gerritsen, "Short-wavelength two-photon excitation fluorescence microscopy of tryptophan with a photonic crystal fiber based light source", Optics Express, 13, 5363-5368 (2005)

[9] C Lefort, L Magnol, P Lévêque, V Blanquet, "Endogenous two-and three-photon fluorescence of a biological substance with a picosecond ultrawide band laser source", Optics, Photonics, and Digital Technologies for Imaging Applications V, $10679 \mathrm{~N}$ (2018)

[10] C. Poudel, C. F. Kaminski, "Supercontinuum radiation in fluorescence microscopy and biomedical imaging applications", JOSA B, 36 (2), A139-A153 (2019)

[11] S. W. Hell, S. J Sahl, M. Bates, X. Zhuang, R. Heintzmann, M. J Booth, J. Bewersdorf, G. Shtengel, H. Hess, P. Tinnefeld, A. Honigmann, S. Jakobs, I. Testa, L. Cognet, B. Lounis, H. Ewers, S. J Davis, C. Eggeling, D. Klenerman, K. I Willig, G. Vicidomini, M. Castello, A. Diaspro, T. Cordes, "The 2015 super-resolution microscopy roadmap", Journal of Physics D: Applied Physics, 48 (44), 143-150 (2015)

[12] H. Hama, H. Kurokawa, H. Kawano, R. Ando, T. Shimogori, H. Noda, K. Fukami, A. Sakaue-Sawano, A. Miyawak, "Scale: a chemical approach for fluorescence imaging and reconstruction of transparent mouse brain". Nature Neuroscience, 14, 1481-1488 (2011)

[13] W. Richardson, "Bayesian-Based Iterative Method of Image Restoration", JOSA 62 (1), 55-59 (1972)

[14]E. Chouzenoux, T.T.K. Lau, C. Lefort, J.-C. Pesquet, “ Optimal Multivariate Gaussian Fitting with Applications to PSF Modeling in Two-Photon Microscopy Imaging”, Journal of Mathematical Imaging and Vision 61 (7), $1037-1050$

[15]D. S. C. Biggs, “3D deconvolution microscopy”, Current Protocols in Cytometry, 52, 12191-1219-20 (2010)

[16] D. A. Fish, A. M. Brinicombre, E. R. Pike, J. G. Walker, "Blind deconvolution by means of the RichardsonLucy algorithm", JOSA A, 12 (1), 58-65 (1995) 